\begin{tabular}{cc}
\hline The Open Urology \& Nephrology \\
Journal \\
CrossMark \\
Content list available at: www.benthamopen.com/TOUNJ/ \\
DOI: $10.2174 / 1874303 \times 01811010014$
\end{tabular}

RESEARCH ARTICLE

\title{
Safety and Viability of Totally Tubeless Ambulatory Percutaneous Nephrolithotomy (APCNL) in the Fast Paced World
}

\author{
BM Zeeshan Hameed, Arun Chawla ${ }^{*}$, Padmaraj Hegde, Avinash Odugoudar and Tirth Vasa \\ Department of Urology, Kasturba Medical College, Manipal Academy of Higher Education, India
}

Received: October 10, 2017

Revised: March 10, 2018

Accepted: March 20, 2018

\begin{abstract}
:
Background:

Percutaneous Nephrolithotomy (PCNL) is the gold standard for endoscopic management of large renal stones. Various modifications have been done to bring down the morbidity of this procedure. Ambulatory PCNL (APCNL) defines PCNL as day-care procedure, avoiding overnight hospital stay which is less than 24 hours. Totally tubeless makes faster recovery without the need for double $\mathrm{J}$ stent or nephrostomy tubes. This study aimed at exploring the feasibility and safety of APCNL in selective patients. It also aimed at improvising the procedure to facilitate early recovery and discharge of patients within $24 \mathrm{hrs}$.
\end{abstract}

\section{Materials ands Methods:}

In this study, 12 patients underwent APCNL under general anaesthesia with endotracheal intubation from April 2016 to March 2017. All the procedures were done by a single surgeon. The inclusion criteria were stone size of less than $2 \mathrm{~cm}$, without any comorbidities, Computed Tomography (CT)/Retrograde Pyelogram(RGP) evidence of normal pyelocalyceal anatomy, patient staying within the radius of $15 \mathrm{~km}$, well informed patient. All patients underwent totally tubeless PCNL i.e. without nephrostomy, DJ stent and catheter. Skin infiltration was given with $0.25 \%$ Bupivacaine. Post operatively analgesia was given on demand (intramuscular Aceclofenac / oral Acetaminophen plus Tramadol). All patients were followed up after 2 weeks.

\section{Results:}

Twelve patients underwent prone PCNL. All patients had single puncture (10 lower calyx, 2 middle calyx), Amplatz size was less than $30 \mathrm{~F}$, size of the stone $(1.3 \mathrm{~cm}$ to $2 \mathrm{~cm})$ with a mean size $-1.7 \mathrm{~cm}$, saline used around $300 \mathrm{ml}$. But, two patients were discharged after $36 \mathrm{hrs}$ and $48 \mathrm{hrs}$ due to pain. None of them returned to hospital with haematuria, pain or urinary tract infection.

\section{Conclusion:}

APCNL is a safe procedure in well informed selective patients. These are the patients with small burden of stone and staying close to the hospital. APCNL reduces the hospital stay, expenses and results in early return to work.

Keywords: Ambulatory, Totally tubeless, Percutaneous nephrolithotomy, APCNL, RIRS, ESWL.

\section{INTRODUCTION}

Ever since the formation of a surgical percutaneous tract to access the anatomy of the pelvicalyceal system of the kidney, it paved way to innovation \& urological advances in the procedure indubitably known as percutaneous nephrolithotomy (PCNL). The contemporary approach is superior to the open approach in terms of morbidity, convalescence, \& cost, thereby substituting open removal of large complex calculi.

PCNL has been the gold standard for the larger stones. There has been a constant debate on the indications for

\footnotetext{
* Address correspondence to this author at the Department of Urology, Kasturba Medical College, Manipal Academy of Higher Education, India, Tel: +91-9741970101; E-mail: chawla.uroarun@gmail.com
} 
PCNL vs retrograde intrarenal surgery(RIRS) vs extracorporeal shockwave lithotripsy (ESWL) for stones in between $1 \mathrm{~cm}$ to $2 \mathrm{~cm} \mathrm{[1].} \mathrm{But,} \mathrm{in} \mathrm{practice} \mathrm{the} \mathrm{decision} \mathrm{for} \mathrm{the} \mathrm{procedure} \mathrm{depends} \mathrm{on} \mathrm{the} \mathrm{anatomy} \mathrm{of} \mathrm{the} \mathrm{pelvicalyceal} \mathrm{system,}$ availability of the instruments, cost of the procedure, desire of the patient and the stone free rates.

In this present era, newer technologies have emerged to reduce the morbidity of PCNL. There are smaller instruments which have led to the practice of traditional PCNL undergoing miniaturisation to mini PNL, ultra-mini PNL and micro-PNL [2]. Similarly the traditional 'tubed' PCNL which is presence of the nephrostomy tube/ double J stent and urinary catheter has been replaced with totally tubeless PCNL. Totally tubeless PCNL denotes the absence of both the nephrostomy tube and double J stent.

The technique evolves from its archaic methods, in order to improve better patient outcomes. Out of the conglomeration of advances, Ambulatory PCNL (APCNL), distinct itself from its predecessor, which required a prolonged hospital stay of 4-6 days due to the practice of inserting a nephrostomy tube, at the conclusion of the procedure, for faster healing of nephrostomy tract, promoting haemostasis, preventing urinary extravasation $\&$ draining infection [3].

This study was conducted primarily to look at the feasibility of Ambulatory PCNL. It also aimed at exploring the various factors which influence the outcome of APCNL in selected patients.

\section{MATERIALS AND METHODS}

This was a prospective study done at our tertiary hospital from April 2016 to March 2017. Out of 682 PCNL done during this period 12 patients were selected for totally tubeless Ambulatory PCNL (APCNL). All the procedures were done by the experienced single surgeon.

The standard protocol was followed, which included careful history and examination of the patients. The investigations in the form of complete blood picture, renal function tests with electrolytes, coagulation profile, urine microscopy with culture and sensitivity were done. Imaging included Ultrasound of the kidney ureter and bladder (KUB), Xray KUB, Contrast enhanced Computed Tomography(CECT), Intravenous Urogram (IVU) and Retrograde Pyelogram (RGP) were tailored as per requirement.

The inclusion criteria comprised of a well-informed patient with no associated clinical co-morbidities; residing within a radius of $15 \mathrm{~km}$ from the hospital, visualised stone size $<2 \mathrm{~cm}$ on imaging like XRAY/CT/IVU, no prior renal or ureteric surgery, CT or RGP revealing normal pelvi-calyceal anatomy (Figs. 1, 2).

Preoperatively, preanesthetic evaluation was done on out patient basis, the factors such as Body Mass Index less than 30 and American society of Anaesthesiologists (ASA) score less than 3, nil comorbidities and mentally sound patients were considered. Patients and their family were explained in the language known to them about the procedure, postoperative care, discharge and the follow-up. They were explained regarding the postoperative analgesia, antibiotic regimen and need for re-procedure in the form of double $\mathrm{J}$ stenting. It was ensured that all the patients have sterile urine.

All patients underwent totally tubeless PCNL i.e. without nephrostomy, DJ stent \& catheter. Preoperative antibiotic was administered an hour before the procedure. All the patients were administered general anaesthesia with endotracheal intubation. They underwent cystoscopy and retrograde pyelogram with $5 \mathrm{~F}$ ureteric catheter insertion. $14 \mathrm{~F}$ urinary Foley catheter was placed. Patients were put on prone position. Under fluoroscopy, puncture to the desired calyx were made using $18 \mathrm{G}$ needle by bulls-eye technique. Schullers metallic guide wire was inserted and central rod was placed. Flexible tip 0.0035F safety guide wire was secured. Using Alkens co-axial metallic dilator, tract was dilated ranging between 26 to 30F. Similar size Amplatz ${ }^{\mathrm{TM}}$ was placed. Amplatz size/ nephrostomy tract was selected as per the dilatation of the calyx in the imaging. Every tract was dilated up to the calyx, not till the infundibulum, to avoid infundibular injury and bleeding Care was taken to avoid more than two punctures. Nephroscopy was done using $20.8 \mathrm{~F}$ mini perc nephroscope or 26F regular nephroscope. Stone was removed intact or fragmented into 2 pieces and removed. Nephroscopy was done to visualise the calyces and pelvis for any bleeding/ perforation/ fragments. If there was no bleeding/ perforation procedure was concluded without any placement of nephrostomy tube/ double J stent. Tract was visualised for any active bleeding while withdrawing the Nephroscope. Using $0.25 \%$ Bupivacaine, skin and the PCNL tract was infiltrated with LA. Skin incision was closed with 2-0 Ethilon suture. Intramuscular Aceclofenac injection or oral Acetaminophen 325mg plus Tramadol 37.5mg combination was given, depending on the pain in the postoperative period. They were followed up after 2 weeks. 


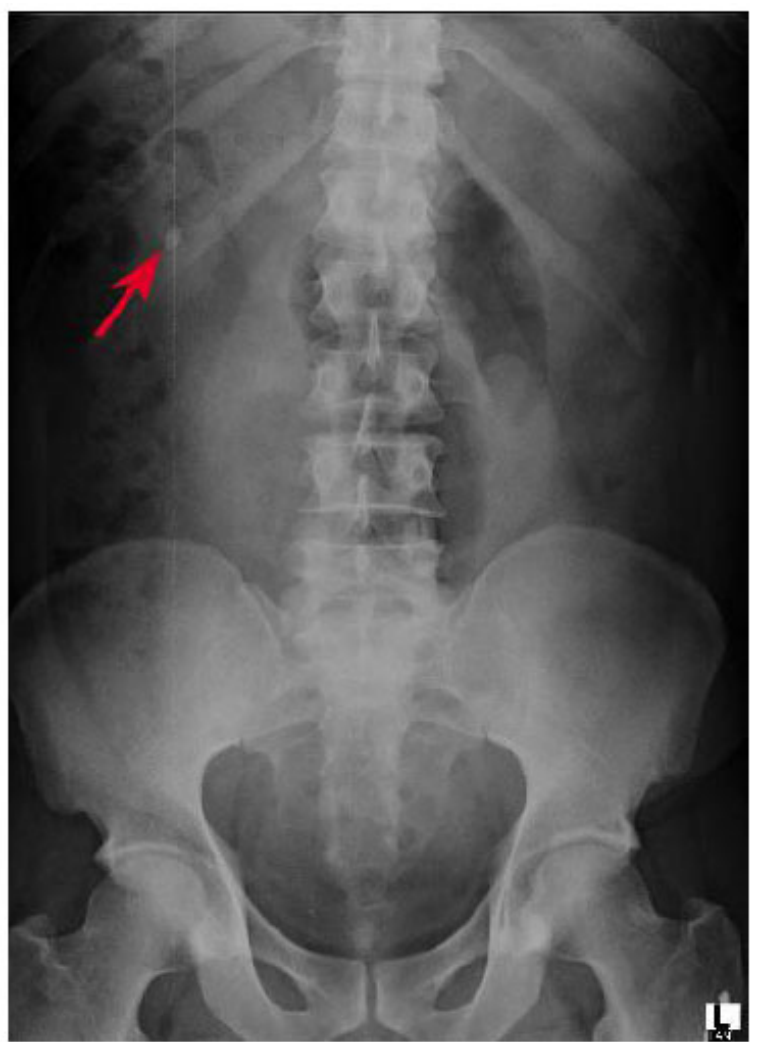

Fig. (1). Xray kidney ureter and bladder (KUB) showing a $1.5 \mathrm{~cm}$ radiopaque shadow at the level of $\mathrm{L}_{1} / \mathrm{L}_{2}$ vertebra in the area of right kidney.

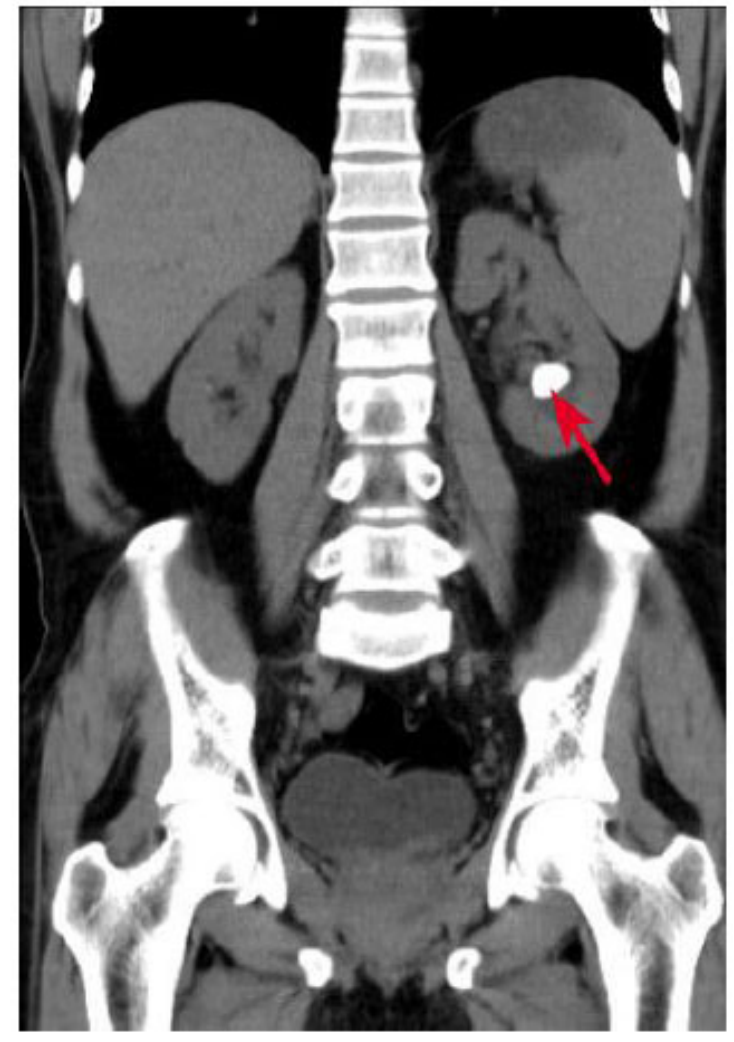

Fig. (2). Non contrast computed tomography (NCCT) KUB showing a $2 \mathrm{~cm}$ calculus in the lower calyx of the left kidney. 


\section{RESULTS}

Twelve patients $(n=12)$ underwent totally tubeless percutaneous nephrolithotmy. Out of 12 patients $n=8(66.6 \%)$ were males and $n=4(33.3 \%)$. The mean age was 44 years with the youngest being 29 years and the other end of spectrum being 55 years. The standard preanesthetic protocol was followed. All were evaluated preoperatively under outpatient basis. Later were given appointment on a planned date. All the patients were admitted on the day of procedure. They underwent prone PCNL under general anaesthesia. The American Society of Anesthesiologists score were 1 in $n=4(33.3 \%)$ patients, 2 in $n=7(58.3 \%)$ patients and 3 in $n=1(8.3 \%)$ patient. Hence more than $90 \%$ of the patients had favourable ASA score (Table 1).

Table 1. Preoperative variables for APCNL cases.

\begin{tabular}{|c|c|c|c|}
\hline Preoperative Criteria & Variable & Number (n=12) & $\mathbf{\%}$ \\
\hline Gender & Male & 8 & 66.6 \\
\hline & Female & 4 & 33.3 \\
\hline Age & Mean \pm S.D & Minimum & Maximum \\
\hline BMI & $44.6 \pm 8.04$ & 29 & 55 \\
\hline Stone Size (cms.) & $26.9 \pm 2.91$ & 22.5 & 31.5 \\
\hline Location & $1.7 \pm 0.21$ & 1.3 & 2 \\
\hline & Upper Calyx & 2 & 0 \\
\hline & Middle Calyx & 7 & 58.3 \\
\hline Lower Calyx & 3 & 25 \\
\hline Side & Pelvis & 6 & 50 \\
\hline LSA Score & Left & 6 & 50 \\
\hline & 1 & 4 & 33.3 \\
\hline Creatinine (mg/dL) & 2 & 7 & 58.3 \\
\hline
\end{tabular}

All patients had single stone. The laterality was equal with $50 \%$ on left and right each. None had stone in the upper calyx. Majority $n=7(58.3 \%)$ had stone in the lower calyx followed by $n=3(25.1 \%)$ in pelvis and $n=2(16.6 \%)$ in the middle calyx (Table 2). The CT imaging showed normal pelvicalyceal anatomy, there were no perinpehric events or anomalies. Out of 12 patients $n=9(74.9 \%)$ had radio-opaque stones, shown on both Xray and CT KUB, $n=3(25.1 \%)$ had radiolucent stones.

Table 2. Stone Characteristics.

\begin{tabular}{|c|c|c|c|}
\hline Stone Characteristics & Variable & Number (n=12) & \% \\
\hline Stone Size (cms.) & $1.7 \pm 0.21$ & 1.3 & 2 \\
\hline Location & Upper Calyx & 0 & 0 \\
\hline & Middle Calyx & 2 & 16.6 \\
\hline & Lower Calyx & 7 & 58.3 \\
\hline Side & Pelvis & 3 & 25 \\
\hline & Left & 6 & 50 \\
\hline
\end{tabular}

After placing the 5Fr ureteric catheter and RGP with visualisation of the pelvicalyceal system and the stone with dilatation of the system, patients were turned prone. All of them $(\mathrm{n}=12)$ had single puncture using the $18 \mathrm{G}$ puncture needle. All had infracostal punctures, with $\mathrm{n}=10(83.3 \%)$ inferior calyceal and $\mathrm{n}=2(16.6 \%)$ middle calyceal punctures. The punctures were clear and were confirmed with the free flow of urine. There was not much of blood staining. The mean fluoroscopy time which was 5 mins. The Amplatz size were as follows: $26 \mathrm{~F}$ were $n=3(25 \%), 28 \mathrm{~F}$ were $\mathrm{n}=$ $4(33.3 \%)$ and $30 \mathrm{~F}$ were $\mathrm{n}=5(46.1 \%)$. The mean operative time was 44.55 mins ( $37 \mathrm{mins}$ to $54 \mathrm{mins}$ ) from cystoscopy to withdrawal of Amplatz sheath. In $n=4$ cases stones were removed intact and in remaining $n=8$ stones were fragmented into two fragments using pneumatic lithotripter and removed. The amount of saline used for irrigation was less than $300 \mathrm{ml}$. None of the patients had any pelvicalyceal bleeding or perforation. Hence, it was totally tubeless 
PCNL without nephrostomy tube or double $\mathrm{J}$ stent. All the patients received local anesthesia at the puncture site with $0.25 \%$ bupivacaine $10 \mathrm{ml}$. skin was closed with the $2-0$ ethilon, which was removed on post op day 7 in the outpatient department (Table 3).

Table 3. Intra-operative variables for APCNL cases.

\begin{tabular}{|c|c|c|c|}
\hline Intraoperative Criteria & Variable & Number $(n=12)$ & $\%$ \\
\hline \multirow[t]{2}{*}{ Puncture Site } & Inferior & 10 & 83.3 \\
\hline & Middle & 2 & 16.6 \\
\hline \multirow[t]{3}{*}{ Amplatz } & $26 \mathrm{~F}$ & 3 & 25 \\
\hline & $28 \mathrm{~F}$ & 4 & 33.3 \\
\hline & $30 \mathrm{~F}$ & 5 & 41.6 \\
\hline \multirow[t]{5}{*}{ Fluoroscopy Time (with Ureteric Catheter insertion \& tract dilatation) (mins.) } & 4 & 2 & 16.6 \\
\hline & 5 & 4 & 33.3 \\
\hline & 6 & 4 & 33.3 \\
\hline & 7 & 2 & 16.6 \\
\hline & Mean \pm S.D & Minimum & Maximum \\
\hline Operating Time (mins.) & $44.5 \pm 5.8$ & 37 & 54 \\
\hline
\end{tabular}

Post procedure patients were taken to the post-operative recovery room. In the recovery room, vitals were checked and saline nebulisation was given for faster recovery from endotracheal tube related convalescence. Once recovered and the urine was clear, the foley catheter was removed. There was negligible drop in the post-operative haemoglobin which was less than $0.1 \%$ (Table 4). Patients were motivated for early mobilisation and hence transferred from the bed to a relaxing chair.

Table 4. Postoperative variables for APCNL cases.

\begin{tabular}{|c|c|c|c|}
\hline Postoperative Criteria & Mean \pm S.D & Minimum & Maximum \\
\hline Hospital Stay (hours) & $20.39 \pm 0.78$ & 18.48 & 22.76 \\
\hline Haemoglobin (g/dL) & $12.47 \pm 0.62$ & 11.8 & 13.6 \\
\hline
\end{tabular}

The mean hospital stay was 20 hours and 39 minutes. Before discharge, an ultrasound KUB was done to look for any perinephric collection/ clots in the kidneys/ residual fragments, which was normal. Patients were explained regarding the possible complications like haematuria, retention of urine, loin pain, fever, chills, voiding symptoms, abdominal distension. Oral analgesia in the form of tramadol $37.5 \mathrm{mg}$ plus acetaminopehen $325 \mathrm{mg}$ was given on demand, the time interval was q8th hourly. All the patients required post-operative analgesia, least was 1 tablet taken by $\mathrm{n}=3$ patients and 8 tablets were the maximum taken by $\mathrm{n}=1$ patient (Table $\mathbf{5}$ ).

Table 5. Postoperative analgesia.

\begin{tabular}{|c|c|c|c|}
\hline Postoperative Analgesia & Mean \pm S.D & Minimum & Maximum \\
\hline & Variable & Number $(\boldsymbol{n}=\mathbf{1 2})$ & \% \\
\hline Analgesia (No. of Oral Acetaminophen \& Tramadol tablets) & 3 & 1 & 8.3 \\
\hline & 4 & 1 & 8.3 \\
\hline & 5 & 3 & 25 \\
\hline & 6 & 3 & 25 \\
\hline & 7 & 3 & 25 \\
\hline
\end{tabular}

All the patients were discharged on the same operative evening, once they were ambulant and the urine was clear. Nevertheless, $\mathrm{n}=2$ patients who were discharged visited the emergency department the next day; $36 \mathrm{hrs}$ and $48 \mathrm{hrs}$ due to loin pain. USG KUB, CT KUB and urine microscopy were done, which were normal. They were given a shot of intramuscular aceclofenac $75 \mathrm{mg}$ injection, with which the pain subsided. Counselling regarding immediate follow up, in case of haematuria, pain or urinary tract infection was done, however none approached with the aforementioned.

\section{DISCUSSION}

The formation of a surgical percutaneous tract to access the anatomy of the pelvi-calyceal system of the kidney was described by Fernstrom \& Johansson (1976) and staged by Wickham (1979). They started with percutaneous nephrostomy under local anaesthesia, followed by the dilatation of the tract serially over the next few days, with 
subsequent stone removal under general anaesthesia using a rigid $30^{\circ}$ cystoscope. This paved way to innovation $\&$ urological advances in the procedure known as Percutaneous nephrolithotomy (PCNL) [4 - 6].

The technique evolves from its archaic methods, in order to excogitate better patient outcomes. The contemporary approach is superior to the open approach in terms of morbidity, convalescence, \& cost, thereby substituting open removal of large complex calculi.

Traditional school of thought was inclined towards standard PCNL, where nephrostomy tubes provided haemostasis along the tract, avoiding urinary extravasation \& maintained adequate drainage from the kidney and the use of double $\mathrm{J}$ stents for urinary drainage down to the bladder [7]. However, based on the concept that the purpose of the tube is only to maintain adequate drainage of the kidney, a 'tubeless' approach has been developed by placing a ureteral stent or catheter to provide drainage after PCNL in lieu of a nephrostomy tube.

Totally tubeless approach was first reported by Wickham and co-workers. They stated that 'provided the kidney is stone-free, the collecting system remains intact and there is not excessive bleeding, there is no need of nephrostomy tube'. This approach was banked upon by Aghamir et al. with patient inclusion criteria comprising of anomalies like horseshoe kidneys, rotational anomalies \& ectopic kidneys [8]. The derivative from the study highlighted the lower analgesic requirements, lesser need for prolonged hospitalisation \& better return to normal day to day life. Totally tubeless PCNL has similar outcomes when compared with standard PCNL in terms of stone-free rate without increasing complications in selected cases. Tubeless PCNL is a safe and effective procedure and is associated with shorter hospitalization and lower analgesic requirement [9]. In selected patients it can be totally tubeless -three tubes less.

Two case series published in 2010, emphasize that with ideal clinical judgement in patient selection, good outcomes are achievable and extending up to a case report published on tubeless PCNL for bilateral struvite stones by Beiko et al and Shahrour et al. Our prospective study, provides quality evidence onto the name of totally tubeless APCNL, in the same manner. In the present study, all the patients $(n=12)$ underwent totally tubeless PCNL without any placement of nephrostomy tube, double J Stent or ureteric catheter. None of these patients, had any intraoperative bleed or injury to the pelvicalyceal system, hence avoiding the tubes.

Appropriate patient selection, plays a crucial basis for attaining the laudative outcome \& ensuring safety of patients in ambulatory PCNL. Residence of a radius of $15 \mathrm{~km}$ along with compliance \& reliability were vital facets of the study. We ensured that the patient understood \& approved the proposed care. Intraoperative all patients, underwent formation of a single tract with less than two punctures and no intraoperative impediments such as excessive haemorrhage or perforation. Upon fluoroscopy, all were deemed to have no stone remnants. In terms of the postoperative criteria, the patients were hemodynamically stable evidenced by normal haemoglobin values.

The first research on APCNL was by Singh et al, who published 10 cases with spinal anaesthesia [10]. The patients were kept for observation for 40 hours. Correspondingly, in another study by Bellman et al, the median hospital stay was 0.6 day or 14.4 hours [11]. Our study demonstrated a shorter median patient hospital stay was less than 24hrs, which was $20.39 \mathrm{hrs}$.

The study done by Beiko et al, had three patients with tubeless PCNL and the mean operating time was 70 mins. They had placed the ureteric catheters which were removed post operatively. In our study the number of patients was 12 and all underwent totally tubeless PCNL without any tubes. The mean operating time was less when compared to their study (44.5 mins vs $70 \mathrm{~min}$ ). However, the hospital stay was lesser in the study done by Beiko et al. [12]

Since the patients get discharged within $24 \mathrm{hrs}$, the probability of repeat visit of patients with complications to the emergency room is high. The usual complications in a patient with totally tubeless PCNL are features of urinary tract infection (UTI), hematuria, sepsis, urinary retention, loin pain. The study done by Shahrour et al had two complications: multiresistant UTI with Escherichia coli. which required higher intravenous antibiotics and the second patient had deep vein thrombosis who received low molecular weight heparin [13]. Our series had no post-operative complications, but two patients returned to the emergency room with loin pain. These patients were evaluated with ultrasound/ CT KUB and urine microscopy which were normal. Hence they were treated with analgesics.

\section{CONCLUSION}

Treatment of renal stone is individualized. Stone up to $2 \mathrm{~cm}$ can be treated by ESWL, RIRS or PCNL, when it is in the lower pole. RIRS involves more cost, may need more than one stage procedure and loss of working days. Any procedure that gives benefit of one stage clearance and early restoration to work with extra advantage of lower costs 
will be suitable for the patients if selected judiciously. Totally tubeless PCNL as done in our patients provides maximum benefits of minimal invasive treatment with lower postoperative pain, early discharge, early reporting to work and no additional procedure (stent removal/ check ureteroscopy) after one month, hence lower the total costs.

By making totally tubeless PCNL ambulatory, the benefits for patients with regard to reduced hospital stay, decreased cost of healthcare and early recovery sums up to the advantage. However, this group of patients should be properly advised and counselled of availing nearby healthcare facility for analgesics, catheterization in case of emergency.

\section{ETHICS APPROVAL AND CONSENT TO PARTICIPATE}

Not applicable.

\section{HUMAN AND ANIMAL RIGHTS}

Not applicable.

\section{CONSENT FOR PUBLICATION}

Consent for publication obtained.

\section{CONFLICT OF INTEREST}

The authors declare no conflict of interest, financial or otherwise.

\section{ACKNOWLEDGEMENTS}

Not applicable.

\section{REFERENCE}

[1] You YD, Kim JM, Kim ME. Comparison of the cost effectiveness of different medical options for treating lower calyceal stones less than 2cm: Extracorporeal shock wave lithotripsy versus percutaneous nephrolithotomy. Korean J Urol 2006; 47(7): 703-7. [http://dx.doi.org/10.4111/kju.2006.47.7.703]

[2] Sabnis RB, Ganesamoni R, Ganpule AP, et al. Current role of microperc in the management of small renal calculi. Indian J Urol 2013; 29(3): 214-8.

[http://dx.doi.org/10.4103/0970-1591.117282] [PMID: 24082443]

[3] Suskind AM, Dunn RL, Zhang Y, Hollingsworth JM, Hollenbeck BK. Ambulatory surgery centers and outpatient urologic surgery among Medicare beneficiaries. Urology 2014; 84(1): 57-61. [http://dx.doi.org/10.1016/j.urology.2014.04.008] [PMID: 24976220]

[4] Fernström I, Johansson B. Percutaneous pyelolithotomy. A new extraction technique. Scand J Urol Nephrol 1976; 10(3): $257-9$. [http://dx.doi.org/10.1080/21681805.1976.11882084] [PMID: 1006190]

[5] Wickham JE, Kellett MJ. Percutaneous nephrolithotomy. Br J Urol 1981; 53(4): 297-9. [http://dx.doi.org/10.1111/j.1464-410X.1981.tb03181.x] [PMID: 7260539]

[6] Ko R, Soucy F, Denstedt JD, Razvi H. Percutaneous nephrolithotomy made easier: A practical guide, tips and tricks. BJU Int 2008; 101(5): 535-9.

[http://dx.doi.org/10.1111/j.1464-410X.2007.07259.x] [PMID: 17922862]

[7] Winfield HN, Weyman P, Clayman RV. Percutaneous nephrostolithotomy: Complications of premature nephrostomy tube removal. J Urol 1986; 136(1): 77-9. [http://dx.doi.org/10.1016/S0022-5347(17)44733-1] [PMID: 3712621]

[8] Aghamir SM, Mohammadi A, Mosavibahar SH, Meysamie AP. Totally tubeless percutaneous nephrolithotomy in renal anomalies. J Endourol 2008; 22(9): 2131-4.

[http://dx.doi.org/10.1089/end.2008.0015] [PMID: 18811568]

[9] Crook TJ, Lockyer CR, Keoghane SR, Walmsley BH. Totally tubeless percutaneous nephroltihtomy. J endourol 2008; $22: 267-71$.

[10] Singh I, Kumar A, Kumar P. “Ambulatory PCNL” (tubeless PCNL under regional anesthesia) - a preliminary report of 10 cases. Int Urol Nephrol 2005; 37(1): 35-7. [http://dx.doi.org/10.1007/s11255-004-6706-9] [PMID: 16132756]

[11] Bellman GC, Davidoff R, Candela J, Gerspach J, Kurtz S, Stout L. Tubeless percutaneous renal surgery. J Urol 1997; 157(5): 1578-82. [http://dx.doi.org/10.1016/S0022-5347(01)64799-2] [PMID: 9112480]

[12] Beiko D, Lee L. Outpatient tubeless percutaneous nephrolithotomy: The initial case series. Can Urol Assoc J 2010; 4(4): E86-90. [http://dx.doi.org/10.5489/cuaj.886] [PMID: 20694090] 
[13] Shahrour W, Andonian S. Ambulatory percutaneous nephrolithotomy: Initial series. Urology 2010; 76(6): $1288-92$. [http://dx.doi.org/10.1016/j.urology.2010.08.001] [PMID: 21130245]

\section{(C) 2018 Hameed et al.}

This is an open access article distributed under the terms of the Creative Commons Attribution 4.0 International Public License (CC-BY 4.0), a copy of which is available at: https://creativecommons.org/licenses/by/4.0/legalcode. This license permits unrestricted use, distribution, and reproduction in any medium, provided the original author and source are credited. 